\title{
Sulla propagazione libera e perturbata delle onde luminose in un mezzo isotropo.
}

\author{
(Memoria di Gisn Antonio MagGi, a Messina.)
}

\begin{abstract}
Supposto un mezzo elastico in vibrazione, senza variazione di densità, indicando con $x, y, z$ e con $x+u, y+v, z+v$ le coordinate di un suo punto qualunque, nello stato d'equilibrio, e alla fine del tempo qualunque $t$, per modo che $u, v, w$ rappresentino le componenti parallele ai tre assi coordinati dello spostamento (estremamente piccolo) del punto, alla fine del tempo $t$, sarà :

$$
\imath=\frac{d V_{2}}{d z}-\frac{d V_{3}}{d y}, \quad v==\frac{d Y_{3}}{d x}-\frac{d V_{1}}{d z}, \quad \vartheta v=\frac{d V_{1}}{d y}-\frac{d V_{2}}{d x},
$$

dove $V_{1}, V_{2}, V_{3}$ indicano tre funzioni (sistema potenziale del movimento), che soddisfamo all' equazione differenziale:

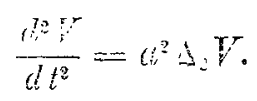

Di tale natura si suppongono le vibrazioni luminose. E la ricerca delle condizioni del mezzo che ne è sede, nell'ipotesi che vi siano immersi una sorgente luminosa, e dei corpi estranei di qualunque forma, col metodo di KincHHOFF, si riduce puramente a quella di un integrale della precedente equazione, che, sulle superfici che limitano il campo occupato dal mezzo, ricevono determinati valori, atti a definire la natura dei corpi estranei rnedesimi. Questo metodo ha quindi il pregio di attirare per la via più diretta e più semplice $\mathbf{i}$ fenomeni relativi alla propagazione perturbata della luce nel dominio della teoria dell' elasticità. Oltre di che le proposizioni analitiche su cui riposa, assai importanti anche per sè stesse, sono suscettibili di una notevole interpretazione fisica: mediante la quale il Principio di Hurgnens, invocato da Hurghens me-
\end{abstract}


desimo in poi per spiegare quei fenomeni, si può considerare come ridotto a più precisi termini, e rigorosamente dimostrato.

Su tale argomento versa uno degli ultimi lavori pubblicati dal compianto Maestro: cioè la Memoria: Zü Theorie der Lichtsstrahlen, inserita nei "Sitzungsberichte " dell' Accademia di Berlino (1882): che apparve ristampata negli "Annalen der Physik und Chemie" (1884), e, tradotta in francese, negli "Annales de l'École Normale Supérieure" (1886, (3) III). Studii nello stesso indirizzo, o intorno ad esso, per quanto mi consta, non furono ancora fatti conoscere da alcuno.

Le seguenti ricerche riguardano il caso generale, e, fra i casi particolari, quello della propagazione della lace perturbata da corpi neri, nell'ipotesi che si reputino insensibili le quantità dell'ordine di grandezza della lunghezza d'onda.

Principale strumento del metodo è una formola, in virtù della quale la funzione cercata $V$ è espressa da un integrale esteso alla superficie del campo considerato, formato coi valori della funzione, e della sua derivata per rispetto alla normale alla superficie, sopra la superficie medesima.

Kirchiof la deduce dalla formola di Green, valendosi di una funzione ausiliaria, la cui possibilità, per le proprietà che si suppone accumulare, sembra possa mettersi in dubbio. Tale difficoltà è evitata dal procedimento ch'jo seguo: alla cui esposizione è dedicata la parte I.

Lo studio dell'integrale che figura nella formola stessa, esteso ad una superficie limitata da un contorno, nell'ipotesi di onde sferiche qualunque, e poi specialmente in quella che siano capaci d'interferire - studio occorrente per l'applicazione della formola ad un campo ove si trovano immersi un punto luminoso e un corpo nero - è oggetto della parte II. A tal fine ricorro alla trasformazione dell' integrale in un integral lineare esteso al contorno della superficie: e le proprietà desiderate ne emergono nel più chiaro modo. Per la trasformazione medesima mi giovo con molto vantaggio delle coordinate sferoidiche, corrispondenti alla determinazione dei punti dello spazio colla mutua intersezione di uno sferoide, di una falda d'iperboloide di rotazione ad esso omofocale, e di un piano terminato alla retta dei fochi comuni alle due quadriche.

La parte III è dedicata ad una dimostrazione della formola di STores, cho serve di base alla suddetta trasformazione in coordinate curvilinee generali.

Finalmente, nella parte IV, sulla traccia di Kinchноғ, considero brevemente il problema della formazione dell' ombra, sufficiente a mostrare l'importanza dei precedenti teoremi. 
I.

$\S 1$. Un notissimo integrale dell' equazione

$$
\frac{d^{2} V}{d t^{2}}=a^{2} \Delta_{2} V
$$

$\grave{\mathrm{e}}$

$$
V=\frac{\varphi\left(t--\frac{r}{a}\right)}{r}
$$

dove, indicando $x_{0}, y_{0}, z_{0}$ le coordinate di un punto qualunque della regione considerata, e $x, y, z$ quelle di un altro punto dello spazio, è:

$$
r=\left|\sqrt{\left(x_{0}-x\right)^{2}+\left(y_{0}-y\right)^{2}+\left(z_{0}-z\right)^{2}}\right| ;
$$

e $\varphi(\xi)$ indica una funzione arbitraria, assoggettata alla sola condizione di ammettere la derivata seconda finita, nell'intervallo corrispondente al campo $\left(x_{0}, y_{0}, z_{0}, t\right)$ nel quale dev'essere soddisfatta la (1).

In questo caso la $V$, data da (2), soddisfarà all'equazione proposta, per ogni valore considerato del tempo $t$, e in tutta la regione voluta, escluso il punto $(x, y, z)$, se, per avventura, vi apparterrà.

§ 2. La funzione $\varphi\left(t-\frac{r}{a}\right)$ potrà contenere $x, y, z$, indipendentemente da $r$ in un modo qualsivoglia: e, per metterlo in evidenza, gioverà indicarla $\operatorname{con} \varphi\left(t-\frac{r}{a}, x, y, z\right)$.

Siano $x, y, z$ le coordinate dei punti di una superficie, la cui area è indicata con $\sigma$, e $f(t, x, y, z), \varphi(t, x, y, z)$ due funzioni di esse e del tempo $t$. Altre soluzioni di (1) saranno:

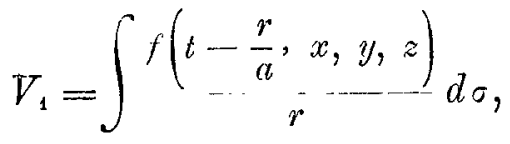

$$
\begin{aligned}
& V_{2}=\int \frac{\partial \frac{\varphi\left(t-\frac{r}{a}, x, y, z\right)}{r}}{\partial n} d \sigma, \\
& V=V_{1} \pm V_{2},
\end{aligned}
$$


dove $r$ è dato da (3): $\frac{d}{d \cdot n}$ indica la derivata per rispetto alia normale $a s$,

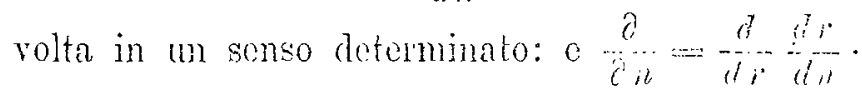

\$ 3. Supposto che $V$ rappresenti la componente di uno spostamento elastico secondo una certa diczione, oppure una delle funzioni del relativo sistema potenziale, la (2) significheià che una serie di onde sforicho emana dal centro d'agitazione $(x, y, z)$, e si propaga nel mezzo, di cui $\left(x_{0}, y_{0}, z\right)$ rappresconta un punto qualunque, con relocita $a: l_{a}(2)_{2}$ e la $(2)_{3}$, che, nel mezzo stesso, si propaga un sistema di tali onde, emansiti da centri d'agitazione distribuiti sula supenficie o, o fomanti, nel primo caso, mo strato semplice, e, nel secondo, un doppio strato. Qucsto, come si rileverì subito, scrivendo

$$
V_{z}=\int_{\Delta=0}^{p\left(t-\frac{1}{a}\left(r+\frac{\Delta r}{\Delta n} \Delta n\right), r, y, z\right) \quad p(t-1+a, x, y, z)}
$$

si deve concepire come formato da un dopyio sistema di centri, distribuiti sulla superficie o, o sopra un'altra che la racchiude, distandone dappertutto infinitamente poco, e tali che da due centri appartenenti alle due superfici, posti sulla stessa normale a $\sigma$, emanano, ad ogni istante, vibrazioni eguali e di senso contrario.

$\S 4$. Colla riflessione che il semplice strato e il doppio strato esauriscono per ora le distribuzioni superficiali di centri d'azione, che serrono alla teoria matematica dei fenomeni fisici, si troverà naturale di assumere (2) quando si voglia ammettere che il mezzo elastico sia in istato di vibrazione per opera di una superficio di centri d'agitazione.

Alla stessa zoluzione si può giungere anche per la via indicata da KIronHOFF nelle sue insigni Vorlesungen ibber Mathematische Physit;, secondo la quale le formole di mecanica trovano la loro ragione nella semplicità e opportunità della corrispondente espressione analitica. A tal fine osserveremo che dalla soluzione più semplice (2), scaturiscono, per la linearitit dell'equazione (1), le soluzioni:

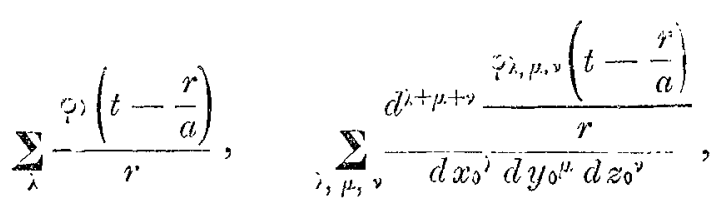


dove $\varphi_{\lambda}(\xi), \varphi_{\lambda, \mu, \nu}(\xi)$ indicano funzioni come $\varphi(\xi)$, variabili per avrentura cogli indici; che se queste somme, col crescere indefinitamente del numero dei loro termini, avranno un limite, questo, sotto le debite condizioni, sarà anche una soluzione di (1). Couformemente a ciò, dalla prima formola, si dedurrà immediatamente $(2)_{2}$ : e $(2)_{3}$, dalla seconda, prendendone la forma:

$$
\begin{gathered}
\frac{d}{d x_{0}} \int \frac{\varphi\left(t-\frac{r}{a}, x, y, z\right)}{r} \frac{d x}{d n} d \sigma+\frac{d}{d y_{0}} \int \frac{\varphi\left(t-\frac{r}{a}, x, y, z\right)}{r} \frac{d y}{d n} d \sigma+ \\
+\frac{d}{d z_{0}} \int \frac{\varphi\left(t-\frac{r}{a}, x, y, z\right)}{r} \frac{d z}{d n} d \sigma .
\end{gathered}
$$

§5. Il nostro ultimo scopo è ora di trovare sotto quali condizioni una espressione come $(2)_{4}$ rappresenterà una determinata funzione, definita in una regione, di cui la superficie $\sigma$ si suppone essere il contorno.

Perciò supponiamo fin d'ora che la superficie $\sigma$ (connessa o no) chiuda una regione, e $x, y, z$ indichino le coordinate di un punto qualunque di essa. Siano poi, per un momento, queste variabili indicate con $x^{\prime \prime}, y^{\prime \prime}, z^{\prime \prime}$ quando entrano in $r$, e diversamente con $x^{\prime}, y^{\prime}, z^{\prime}$, per modo che

$$
\frac{d x^{\prime}}{d \xi}=\frac{d x^{\prime \prime}}{d \xi}=\frac{d x}{d \xi} ; \quad \frac{d}{d x}=\frac{d}{d x^{\prime}}+\frac{d}{d x^{\prime \prime}} .
$$

E posto simbolicamente, per brevità di scrittura,

$$
F(x)+F(y)+F(z)=[F(x)]
$$

in conseguenza di che

$$
V_{2}=\int\left[\frac{d \frac{\left(t-\frac{r}{a}, x^{\prime}, y^{\prime}, z^{\prime}\right)}{r}}{d x^{\prime \prime}} \frac{d x^{\prime}}{d n}\right] d \sigma,
$$

supponiamo $\varphi(t, x, y, z)$ definita nella regione limitata da $\sigma:$ e $f(t, x, y, z)$, funzione dei punti della superficie stessa, collegata con essa in modo che sia:

$$
V_{\mathrm{i}}=\int\left[\frac{d \frac{\varphi\left(t-\frac{r}{a}, x^{\prime}, y^{\prime}, z^{\prime}\right)}{r}}{d x^{\prime}} \frac{d x^{\prime}}{d n}\right] d \cdot \sigma .
$$

Annali di Matematica, tomo XVI. 
Perciò, basterà ammettero che $\varphi(t, r, y, z)$ ammetta, sopra $\sigma$, le derivate parziali, o porre, sulla superficie stessa:

$$
f(t, x, y, z)=\frac{d \rho(t, x, y, z)}{d \|} .
$$

Posto finalmente:

$$
\begin{aligned}
& \frac{\left(1-\frac{r}{d} \cdot x^{\prime} \cdot y, z^{\prime}\right)}{d \cdot r}+\frac{\left(1-\frac{r}{a}, x^{\prime}, y^{\prime}, z^{\prime}\right)}{d x^{\prime \prime}}=X, \\
& \frac{\partial\left(t-\frac{v}{a}, x^{\prime}, y^{\prime}, z^{\prime}\right)}{d y^{\prime}}=\frac{\partial\left(t-\frac{v}{a}, x^{\prime}, y^{\prime}, z^{\prime}\right)}{d \cdot \ldots \pm \frac{v^{\prime}}{d y^{\prime \prime}}}=Y
\end{aligned}
$$

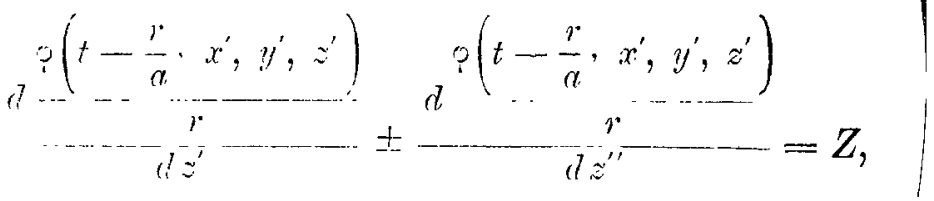

no rerrì, nolle fatte ipotesi,

$$
V=\int\left(X \frac{d x}{d ! n}+Y \frac{d y}{d n}+Z \frac{d z}{d n}\right) d \sigma .
$$

$X, Y, Z$ rappresuntorano in questo integrale $\mathrm{i}$ valori sulla superficie $\sigma$ di tre funzioni definite in tutia la regione limitata da essa. Supponiamo che, nolla regione stessa, queste tie funzioni siano monodrome continue e finite, e dotate ciella derivata parziale finita per rispetto a $x$, a $y$ e a $z$. Perciò basterà che, nella regiono in discorso, $\varphi(t, x, y, z)$ possieda le stesse proprietà, e sia inoltre dotata dolie derivate parziali seconde finite per rispetto a ciascuna variabile due rolte: $e$, in secondo luogo, che alla regione non appartenga il punto $\left(x_{0}, y_{0}, z_{0}\right)$, per modo che sia sempre $r>0$.

In tali ipotesi, per un noto teorema, indicando con $S$ il volume dello spazi chinso da $\sigma$, sarà, per $(5)$ :

$$
V=\int\left(\frac{d X}{d x}+\frac{d Y}{d y}+\frac{d Z}{d z}\right) d S .
$$

$\S$ 6. Procuriamo ora di determinare le condizioni sotto le quali sarà 
in tutta la regione $S$ :

$$
\frac{d X}{d x}+\frac{d Y}{d y}+\frac{d Z}{d z}==0
$$

Dalle (4), prendendo il segno - , e riducendo i termini simili, si ha:

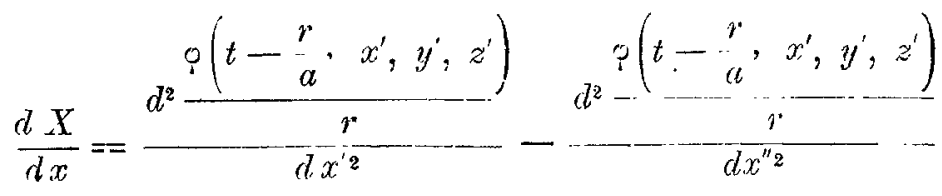

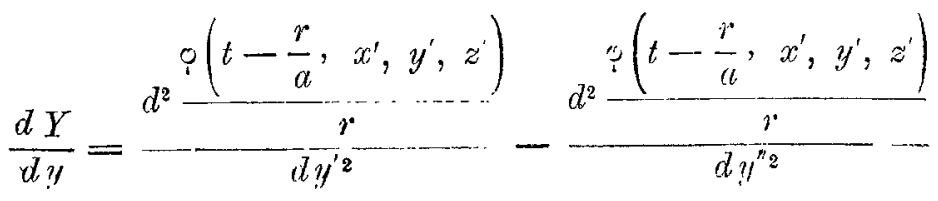

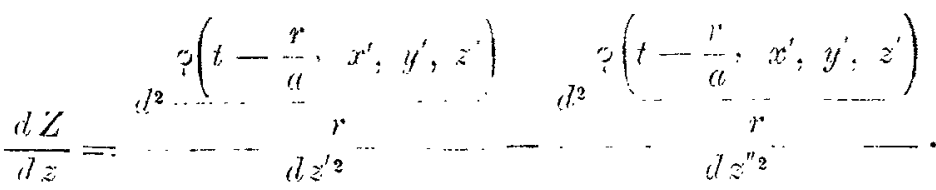

Quindi:

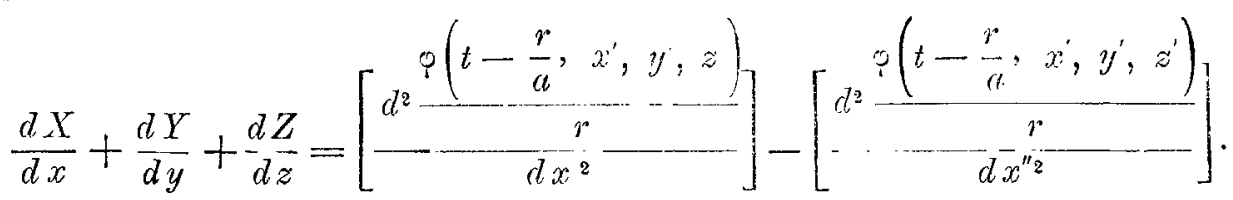

Ora:

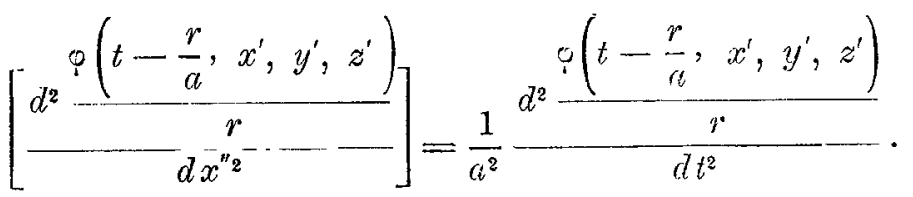

E da ciò segue che, perchè si verifichi la (6), sarà necessario e sunficiente che la funzione $\varphi(t, x, y, z)$ soddisfaccia all' equazione differenziale (1), dove il $\Delta_{2}$ s'intenda riferito alle variabili $x, y, z$. Poichè, siccome $r$ non dipendo da $x^{\prime}, y^{\prime}, z^{\prime}$, si ha:

e se sarà:

$$
\left[\frac{d^{2} ?\left(t, x^{\prime}, y^{\prime}, z^{\prime}\right)}{d x^{2}}\right]=r\left[\frac{d^{2} \frac{\left.p^{t}, x^{\prime}, y^{\prime}, z^{\prime}\right)}{r}}{d x^{\prime 2}}\right]
$$

$$
\left[\frac{d^{2} \varphi\left(t, x^{\prime}, y^{\prime}, z^{\prime}\right)}{d x^{2}}\right]==\frac{1}{a^{2}} \frac{d^{2} ?\left(t, x^{\prime}, y^{\prime}, z^{\prime}\right)}{d t^{2}}
$$


sarà anche:

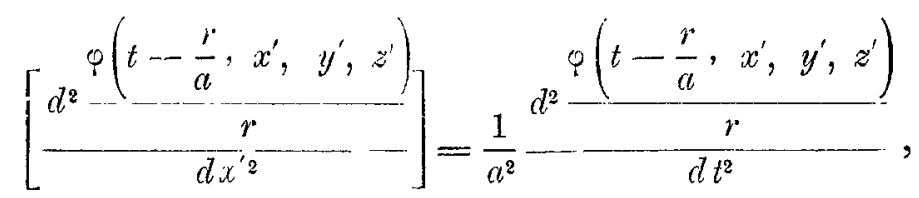

donde per (7), sarì soddisfatta la (6), c. v. d.

$\S 7$. Concludiamo che, data una superficie chiusa (connessa o no) $\sigma$, indicando con $\varphi(t, x, y, z)$, o più brevemente con $\varphi(t)$, una funzione, che, per ogni valore di $t$, e in ogni posto $(x, y, z)$ della regione limitata dalla superficie, esclusi per arventura gli stessi suoi punti, soddisfa all' equazione differenziale (1), posto

e inoltre

$$
r=\sqrt{\left(x_{0}-x\right)^{2}+\left(y_{0}-y\right)^{2}+\left(z_{0}-z\right)^{2}}
$$

$$
\frac{d \varphi(t)}{d n}=f(t), \quad \frac{\partial \frac{\varphi\left(t-\frac{r}{a}\right)}{r}}{\partial n}-\frac{f\left(t-\frac{r}{a}\right)}{r}=\Omega
$$

dove $\frac{\partial}{\partial n}=\frac{d}{d r} \frac{d r}{d n}$, nell'ipotesi che il punto $\left(x_{0} y_{0} z_{0}\right)$ non appartenga alla regione limitata da $\sigma$, nè cada sopra di essa, sarà:

$$
\int \Omega d \sigma=0
$$

$\S 8$. Un ben noto procedimento ci permette di dedurre da questo risultato, già per sè stesso importante, quello a cui principalmente miravamo (cfr. $\S 5)$. Supposto il punto $\left(x_{0} y_{0} z_{0}\right)$ appartenente alla regione limitata da $\sigma$, e precisamente un punto qualunque interno della regione stessa, potremo applicare la (9) alla regione limitata dalla stessa $\sigma$, e internamente dalla superficie di una sfera avente per centro quel punto e raggio comunque piccolo: per modo che, indicando con 5 la superficie di questa sfera, sarà:

$$
\int \Omega d \sigma=-\int \Omega d s \text {. }
$$

Si troverà subito che, collo svanire del raggio della sfera, $\int \Omega d \sigma$ ha per limite $-4 \pi \varphi\left(t, x_{0}, y_{0}, z_{0}\right)$, che per breviti indicheremo con $-4 \pi \varphi_{0}(t)$ : e per conseguenza, eguagliando $i$ limiti dei due membri della precedente eguaglianza:

$$
\int \Omega d \sigma=4 \pi \varphi_{0}(t)
$$




\section{Questo risultato e quello del precedente paragrafo si potranno compendiare in una formola, scrivendo:

$$
\int \Omega d \sigma=4 \pi \varepsilon \varphi_{0}(t)
$$

dove $\varepsilon=1$ oppure $\varepsilon=0$, secondo che il punto $\left(x_{0} y_{0} z_{0}\right)$ appartiene o no alla regione di cui $\sigma$ è il contorno.

§ 9. Con analogo artifizio, nell'ipotesi che la regione considerata sia da $\sigma$ internamente limitata e del resto infinita, applicheremo in primo luogo il risultato testè ottenuto ad una regione limitata da $\sigma$ all' interno, e all'esterno dalla superficie di una sfera avente per centro il punto $\left(x_{0} y_{0} z_{0}\right)$ e raggio comunque grande, $\rho$. Indicando la superficie di questa sfera con 5 , e con $\omega$ quella della superficie concentrica di raggio unitario, troveremo facilmente:

$$
\int \Omega d \varsigma=\int_{0}^{4 \pi}\left\{\varphi\left(t-\frac{\rho}{a}\right)-\rho\left(\frac{d \varphi\left(t-\frac{\rho}{a}\right)}{d \rho}\right)+f\left(t-\frac{\rho}{a}\right)\right\} d \omega .
$$

E per conseguenza, se, col crescere indefinitamente di $\rho$, questa quantità avrà per limite 0 , ciò che dipenderà dalla natura della funzione $\varphi(t, x, y, z)$, alla regione in discorso sarà egualmente applicabile la (10) (*).

(*) KIrch hoff, stabilita la (10), procede in un modo che sembra escludere ogni restrizione, osservaudo che, purchè si ammetta che, anteriormente ad un certo valore del temno, a qualunque epoca, tutto lo spazio fusse in quiete: col crescere indefinitamente di $t$, per valori negatiri, $f(t)$ e $\varphi(t)$, in qualunque posto, comunqua lemoto si concepisea, avranno per limite 0 : e quindi, col creseere indefinitamente di $p$, arranno per limite 0 anche $\varphi\left(t-\frac{\rho}{a}\right)$. $f\left(t-\frac{p}{a}\right)$, e cinalmente $\int \boldsymbol{\Omega} d s$.

Ora, per poter scrivere l'equazione limite della (10), o, coi termini di Kirchroff, applicarla al valore infinito di $\rho$, bisogna prima ch'essa sussista comunque grande si concepisca $p$, ossia grande in valore assoluto $t-\frac{p}{a}$. E poichè l'equazione stessa suppone che la furzione $\rho_{i}(t, x, y, z)$, in tutto lo spazio, e per tutti i valori del tempo che occorre considerare, sia finita e continua, ammetta le derivate fornite delle stesse proprieta, e soddisfaccia alla (1), per applicare legittimamente il metodo anzidetto, attribuita a $\varphi(t, \infty, y, z)$ una forma, tra i limiti del tempo che corrispondono al fenomeno considerato, bisorna dimostrare, o ammettere. l'esistenza di una funzione, che coincide con essa fra quei limiti, riceve il valore 0 in ogni punto, per $t$ algebricamente inferiore ad un certo valore, e tuttavia non cessa per nessun valore del tempo, e in nessun punto, di possedere le precedenti proprieta.

Inoltre può suscitare obliezioni la stessa ipotesi fundamentale, che obbliga a introdurre Je condizioni dello spazio, fuori dei limiti naturali prescritti dal fenomeno. Mentre, nel caso nostro, occorre appena di osservare che il risultato analitico sta, si vorlia ammettere o no che, fuori di quei limiti, la funzione $\varphi(t, x, y, z)$ conservi lo stesso significato meccanico. 
$\S 10$. Le condizioni preseritte per (10) sono soddisfatte dall'ipotesi:

$$
\varphi(t, x, y, z)=\frac{\dot{\varphi}\left(r_{1}-a t\right)}{r_{1}},
$$

indicando con $\psi(\xi)$ una funzione arbitraria, dotata, in un opportuno intervallo, della derivata seconda finita, e posto:

$$
r_{1}=\sqrt{ }\left(x-x_{1}\right)^{2}+\left(y-y_{1}\right)^{2}+\left(z-z_{1}\right)^{2},
$$

dove $x_{1}, y_{1}, z_{1}$ rappresentano le coordinate di un punto fisso, non appartenente alla l'egione di cui $\sigma$ è il contorno.

In questo caso,

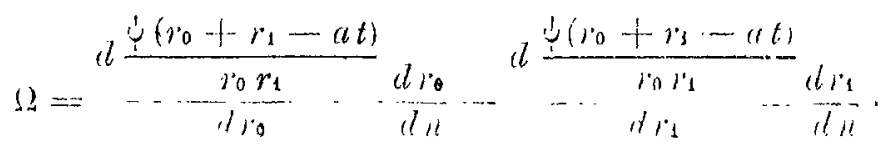

L nolle ilmerei del $\$ 8$, posto inoltre:

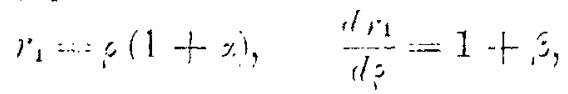

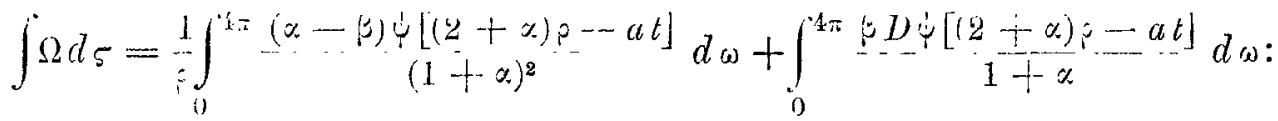

quantità che, col crescere di $\rho$, in conseguenza di che $\alpha$ e $\beta$ tendono a 0 , ha per limite 0 ; donde si conclude, per lo stesso $\S 8$, che la formola (10) si applichcrà anche ad una regione internamente limitata da $\sigma$ e del resto infinita.

$\S 11$. Supponiamo che il punto $\left(x_{1} y_{1} z_{1}\right)$ si trovi nell' interno della regione limitata (per avventura interiormente) da $\sigma$. Coll'artifizio sopra ricordato, applicando in primo luogo la (10) alla regione egualmente limitata, e avente inoltre per contorno interno la superficie di una sfera con centro nel punto $\left(x, y_{1} z_{1}\right)$, e raggio comunque piccolo: e eguagliando poi i limiti dei due membri dell'eguaglianza collo svanire di questo raggio, ne dedurremo:

$$
\int \Omega d \sigma=4 \pi \varepsilon^{\prime} \varphi_{0}(t)
$$

dove $\varepsilon^{\prime}=-1$, oppure $\varepsilon^{\prime}=0$, secondo che la regione considerata contiene 0 no, oltre il punto $\left(x_{1} y_{1} z_{1}\right)$, il punto $\left(x_{0} y_{0} z_{0}\right)$.

$\mathrm{E}$ intendendo che sia $\varepsilon^{\prime}=0$, se la regione di cui $\sigma$ è il contorno non contiene alcuno dei punti $\left(x_{0} y_{0} z_{0}\right),\left(\begin{array}{lll}x_{1} & y_{1} & z_{1}\end{array}\right)$, oppure li contiene ambedue, $\varepsilon^{\prime}=1$, se contiene soltanto il primo, $\varepsilon^{\prime}=-1$, se contiene soltanto il secondo, la (10) compendierà i risultati di questo paragrafo e del precedente. 
$\S$ 12. Di questi risultati è ovvia l'interpretazione meccanica. Essi significano che, supposto un mezzo elastico, omogeneo, in ribrazione per opera di un centro, l'azione di questo sopra ogni punto del mezzo sarà equivalente a quella di uno strato e di un doppio strato di centri, distribuiti sopra una superficie qualunque, racchiudente il centro agitatore, e non il punto considerato. Che se si considera un punto del mezzo egualmente racchiuso da quella superficie, l'azione sopra di esso del sistema formato dallo strato e dal doppio strato sarà nulla.

E queste sono in sostanza le condizioni supposte dal celebre Principio di Huyghens.

$\S$ 13. Rappresentiamo finalmente con $\varphi^{*}(t, x, y, z)$, o con $\varphi^{*}(t)$, la soluzione particolare di (1) ora considerata: e con $\varphi(t, x, y, z)$, o con $\varphi(t)$, un'altra soluzione qualunque di essa, colla sola restrizione che la differenza $\varphi(t, x, y, z)-\varphi^{*}(t, x, y, z)$ non presenti eccezioni in alcun punto della regione limitata da $\sigma$.

Indicando questa differenza con $\delta \varphi^{*}(t, x, y, z)$ o con $\delta \varphi^{*}(t)$, e con $\Omega^{*}$, $\delta \Omega^{*}$, le espressioni come $\Omega$, corrispondenti a $\varphi^{*}(t)$ e $\delta \varphi^{*}(t)$, anzichè a $\varphi(t)$, si avrà, in virtì di $(10)$, per ogni punto $\left(\begin{array}{llll}x_{0} & y_{0} & z_{1}\end{array}\right)$ interno alla regione di cui $\sigma$ ò il contorno:

$$
4 \pi \delta^{2} \varphi_{0}^{*}(t)=\int \delta \Omega^{*} \cdot d \sigma
$$

dove $\delta \varphi_{0}^{*}(t)=\delta \varphi^{*}\left(t, x_{0}, y_{0}, z_{0}\right)$; e supposto il punto $\left(x_{1} y_{1} z_{1}\right)$ interno alla regione medesima, in virtù di $(11)$,

$$
\int \Omega^{*} d \sigma=0
$$

per modo che, nella stessa ipotesi:

e finalmente:

$$
4 \pi \delta \varphi_{0}^{*}(t)=\int\left(\Omega^{*}+\delta \Omega^{*}\right) d \sigma=\int \Omega d \sigma
$$

$$
4 \pi \varphi_{0}(t)=4 \pi \varphi_{0}^{*}(t)+\int \Omega d \sigma
$$

formola che, purchè la funzione $\varphi(t)$ soddisfaccia alla condizione a suo luogo indicata $(\S 9)$, si potrà applicare ad una regione da $\sigma$ internamente limitata, e del resto infinita.

Supponiamo, per fissare le idee, quest' ultimo caso. Allora, perchè $\int \varrho d \sigma$ abbia un valore diverso da 0 , sarà necessario, per $(10)$, che, nella regione chiusa 
dalla superficie $\sigma$, o da parte di essa, e cioè di cui $\sigma$, o parte di essa, è il contorno completo, la funzione $\varphi(t)$ cessi di essere definita, o per lo meno presenti qualche eccezione alle proprietà che le sono supposte nella regione internamente limitata da $\sigma$, e quindi fuor $i$ delle regioni predette.

$\mathrm{Ne}$ viene che l'equazione precedente conviene all'ipotesi che nel mezzo considerato si trovino, oltre il centro agitatore, dei corpi estranei (rappresentati da quelle regioni), i quali ne perturbano l'azione.

E l'azione di questi corpi risulta equivalente a quella di uno strato e di un doppio strato di centri distribuiti sulla loro superficie, o sopra una superficie che gl'involge: ciò che è pure conforme al Principio di Huyaness.

II.

$\S$ 1. La teoria, conforme ai precedenti principii, della propagazione delle onde luminose emananti da un centro, in un mezzo occupato da corpi neri, si fonda sulle proprietà di $\int \Omega d \sigma$, dove si suppone, come nel $§ 10$ di I,

$$
\varphi(t, x, y, z)=\frac{\psi\left(r_{1}-a t\right)}{r_{1}},
$$

e la superficie d'area $\sigma$, alla quale s'intende esteso l'integrale, limitata da un contorno.

Gioverà quindi premettere lo studio di questo integrale: e, prima di tutto, qualche considerazione sulle coordinate curvilinee, di cui per quello studio ci vagliamo.

2. Posto:

$$
r_{0}+r_{1}=2 \zeta, \quad r_{0}-r_{1}=2 \theta,
$$

dove $r_{0}, r_{1}$ indicano la distanza di un punto qualunque dello spazio da due punti fissi, che chiameremo il punto 0 e il punto 1 , sarà, in primo luogo, indicando con $c$ la semi-distanza mutua dei due punti fissi

Di qui

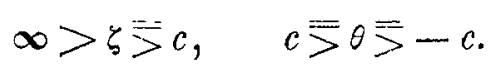

$$
\zeta^{2} \geq c^{2} \geq \theta^{2}
$$

Inoltre, sulla retta passante pei due punti fissi 0,1 :

$$
\begin{aligned}
& \zeta^{2}=c^{2}=\theta^{2} \quad: \text { nei due punti, } \\
& \zeta^{2}=c^{2}, \quad \zeta^{2}>\theta^{2}: \text { nel segmento } 01, \\
& \zeta^{2}>\theta^{2}, \quad \sigma^{2}=c^{2}: \text { fuori del segmento } 01 .
\end{aligned}
$$


$\zeta$ rappresenta il semi-asse (polare) di un ellissoide di rotazione avente per fochi i punti 0 e 1: $|\theta|$ il semi-asse di un iperboloide di rotazione a due falde, avente per fochi gli stessi punti. Ed essendo $\theta>0$ oppure $\theta<0$, secondo che $r_{0}>r_{1}$ oppure $r_{0}<r_{1}$, ad ogni valore di $\theta$ corrisponde una falda dell' iperboloide: quella che chiude il punto 0 , oppure quella che chiude il punto 1 , secondo che è $\theta<0$ o $\theta>0$.

Indichi w l'angolo formato da un piano limitato alla retta dei punti 01 , con un piano simile fisso.

Ad ogni terna di valori $\zeta, \theta$, $\omega$ delle quantità cos̀̀ definite corrisponderà un punto, ed uno solo, dello spazio, definito come intersezione delle relative superficie delle tre diverse famiglie. Mentre, reciprocamente, per ogni punto dello spazio passa una sola superficie di ciascuna delle tre famiglie: per modo che vi corrisponderà una terna, ed una sola, dei predetti valori.

$\S 3$. ¿ cresce col passare da uno sferoide ad un altro che l'abbraccia: e perciò, lungo il mezzo ramo iperbolico corrispondente a determinati valori di $\omega$ e di $\theta$, partendo dal vertice.

$|\theta|$ cresce, col passare da una falda iperbolica ad un'altra da essa abbracciata Quindi $\theta$, tenuto calcolo del segno, cresce a questo modo nella successione delle falde iperboliche che abbracciano il punto 1 , e col passare da una falda ad un'altra che l'abbraccia, nella successione di quelle che chiudono il punto 0: donde segue che, lungo la mezza ellisse corrispondente a determinati valori di $\omega$ e di $\zeta$, crescerà partendo dal vertice più vicino al punto 0 .

Finalmente, w crescerà lungo la circonferenza, corrispondente a determinati valori di $\zeta$ e di $\theta$, nel senso in cui, partendo dal piano fisso, cresce l'angolo così indicato: senso, che è in nostro arbitrio di fissare.

$\$ 4$. La teoria delle coordinate ellittiche nel piano ci dispensa dal trattenerci su quella, affatto analoga, delle coordinate nello spazio in discorso che potremo chiamare sferoidiche. Da quella teoria si deduce immédiatamente che le coordinate stesse sono ortogonali: e che, rappresentando $d s$ il differenziale di un arco di linea, corrispondente ai differenziali $d \zeta, d \theta$ e $d \omega$, sarà:

$$
d s^{2}=\frac{\zeta^{2}-\theta^{2}}{\zeta_{2}-c^{2}} d \zeta^{2}+\frac{\zeta^{2}-\theta^{2}}{c^{2} \cdots \theta^{2}} d \theta^{2}+\frac{\left(\zeta^{2}-c^{2}\right)\left(c^{2}-\theta^{2}\right)}{c^{2}} d \omega^{2}:
$$

altra proprietà, che, come le precedenti, ci occorre avere presente.

$\S 5$. Ciò premesso, consideriamo l'integrale $\int \Omega d \sigma$ di cui al $\S 1$. Noi possiamo concepirlo posto nella forma indicata da (5) di I; e pel $\S 6$ dello Annali di Matematica, tumo XVI. 
stesso $I$, in ogni campo non comprendente i punti singolari 0 e 1 , si verifcherà (6). Ne viene che, sotto le debite condizioni, si potranno determinare $X^{\prime}, Y^{\prime}, Z^{\prime}$ in modo che si verifichino le relazioni:

$$
X=\frac{d Z^{\prime}}{d y}-\frac{d Y^{\prime}}{d z}, \quad Y=\frac{d X^{\prime}}{d z}-\frac{d Z^{\prime}}{d x}, \quad Z=\frac{d Y^{\prime}}{d x}-\frac{d X^{\prime}}{d y} ;
$$

in seguito a che l'integrale si potrà trasformare in un integrale esteso al contorno di $s$, col teorema di Srokes, di cui giova rammentare il seguente enunciato, relativo al caso di coordinate ortogonali qualunque: "Rappresentando $q_{1}, q_{2}, q_{3}$ le coordinate di un punto dello spazio, comunque definite, purchè, pel differenziale $d s$ di un arco di linea, corrispondente agl' incrementi $d q_{1}$, $d q_{2}, d q_{3}$, si abbia:

$$
d s^{2}=Q_{1}^{2} d q_{1}^{2}+Q_{2}^{2} d q_{2}^{2}+Q_{3}^{2} d q_{3}^{2},
$$

(per modo che le coordinate in discorso dovranno essere ortogonali): e con $U_{1}, U_{2}, U_{3}$ tre funzioni di esse, - nell' ipotesi che queste funzioni, come pure $Q_{1}^{2}, Q_{2}^{2}, Q_{3}^{2}$, sulla superficie $\sigma$ siano monodrome, continue e finite e queste tre ultime non si annullino in alcun punto, - sarà in generale:

$$
\left.\begin{array}{c}
\int\left\{\left(\frac{d U_{3}}{d q_{2}}-\frac{d U_{2}}{d q_{1}}\right) \frac{Q_{1}}{Q_{2} Q_{3}} \frac{d q_{1}}{d n}+\left(\frac{d U_{1}}{d q_{3}}-\frac{d U_{3}}{d q_{1}}\right) \frac{Q_{2}}{Q_{3} Q_{1}} \frac{d q_{2}}{d n}+\right. \\
\left.\left.\frac{d U_{2}}{d q_{1}}-\frac{d U_{1}}{d q_{2}}\right) \frac{Q_{3}}{Q_{1} Q_{2}} \frac{d q_{3}}{d n}\right\} d \sigma= \pm \int\left(U_{1} d q_{1}+U_{2} d q_{2}+U_{3} d q_{3}\right) ;
\end{array}\right\}
$$

dove $\frac{d}{d n}$ rappresenta, al solito, il coefficiente differenziale per rispetto alla normale alla superficie: l'integrale del secondo membro va esteso al contorno di $\sigma: Q_{1}, Q_{2}, Q_{3}$ indicano valori assoluti: e il segno + va ad esso applicato, quando, immaginando descritte, per un punto qualunque della superficie $\sigma$, la normale, nel senso in cui si suppone volta per derivare, e le tangenti alle linee coordinate passanti per esso punto, nel senso in cui le coordinate rispettive crescono, quel senso della normale, e quello in cui si percorre il contorno per integrare, saranno, l'uno per rispetto all'altro, come il senso della tangente alla linea $q_{1}$, e quello in cui deve girare un raggio, per passare, dalla tangente alla $q_{2}$, alla tangente alla linea $q_{3} "\left(^{*}\right)$.

$\S 6$. L'accennata trasformazione si ottiene assai facilmente, valendosi delle coordinate sferoidiche.

(*) Tedasi III. 
Per mezzo delle (2), posto, per brevità :

$$
\psi\left(r_{0}+r_{1}-a t\right)=\chi\left(\frac{r_{0}+r_{1}}{2}\right)
$$

si deduce da (11) di I, dopo alcune ovvie riduzioni,

$$
\Omega=\frac{d \frac{\chi(\zeta)}{\zeta^{2}-\theta^{2}}}{d \zeta} \frac{d \theta}{d n}+\frac{d \frac{\chi(\zeta)}{\zeta^{2}-\theta^{2}}}{d \theta} \frac{d \zeta}{d n}
$$

donde, con scrittura appropriata al nostro scopo, essendo, nel caso delle coordinate sferoidiche, con $q_{1}=\theta, q_{2}=\omega, q_{3}=\zeta$, per $(4)_{1}$, e $(4)_{2}$ :

$$
\frac{Q_{1}}{Q_{2} Q_{3}}=\frac{c}{c^{2}-\theta^{2}}, \quad \frac{Q_{2}}{Q_{3} Q_{1}}=\frac{1}{c\left(\zeta^{2}-\theta^{2}\right)}, \quad \frac{Q_{3}}{Q_{1} Q_{2}}=\frac{c}{\zeta^{2}-c^{2}},
$$

in primo luogo:

$\Omega=\frac{1}{c}\left\{\left(\frac{d \frac{c^{2} \chi(\zeta)}{\zeta^{2}-\theta^{2}}}{d \zeta}-\frac{d \frac{\theta^{2} \chi \zeta}{\zeta^{2}-\theta^{2}}}{d \zeta}\right) \frac{c}{c^{2}-\theta^{2}} \frac{d \theta}{d n}+\left(\frac{d \frac{\zeta^{2} \chi(\zeta)}{\zeta^{2}-\theta^{2}}}{d^{\theta}}-\frac{d \frac{c^{2} \chi(\zeta)}{\zeta^{2}-\theta^{2}}}{d \theta}\right) \frac{c}{\zeta^{2}-c^{2}} \frac{d \zeta}{d n}\right\}:$

ed essendo:

per modo che:

$$
\frac{\zeta^{2} y(\zeta)}{\zeta^{2}-\theta^{2}}=\chi(\zeta)+\frac{\theta^{2} \chi(\zeta)}{\gamma^{2}-\theta^{2}}
$$

$$
\frac{d \frac{\theta^{2} \chi(\zeta)}{\zeta^{2}-\theta^{2}}}{d \theta}=\frac{d \frac{\zeta^{2} \chi(\zeta)}{\zeta^{2}-\theta^{2}}}{d \theta}
$$

in secondo luogo:

$\Omega=\frac{1}{c}\left\{\left(\frac{d \frac{c^{2} \chi(\zeta)}{\zeta^{2}-\theta^{2}}}{d_{\zeta}^{\prime}}-\frac{d \frac{\theta^{2} \chi(\zeta)}{\zeta^{2}-\theta^{2}}}{d \zeta}\right) \frac{c}{c^{2}-\theta^{2}} \frac{d \theta}{d n}+\left(\frac{d \frac{\theta^{2} \chi(\zeta)}{\zeta^{2}-\theta^{2}}}{d \theta}+\frac{d \frac{c^{2} \chi(\zeta)}{\zeta^{2}-\theta^{2}}}{d \theta}\right) \frac{c}{\zeta^{2}-c^{2}} \frac{d \zeta}{d n}\right\}$.

Questa formola può scriversi così:

$$
\begin{aligned}
\Omega= & \frac{1}{c}\left\{\left(\frac{d}{d \omega} \frac{d \frac{c^{2} \chi(\zeta) \omega}{\zeta^{2}-\theta^{2}}}{d \zeta}-\frac{d \frac{\theta^{2} \chi(\zeta)}{\zeta^{2}-\theta^{2}}}{d \xi}\right) \frac{c^{2}}{c^{2}-\theta^{2}} \frac{d \theta}{d n}+\right. \\
& \left.+\left(\frac{d \frac{\theta^{2} \chi(\zeta)}{\zeta^{2}-\theta^{2}}}{d \theta}-\frac{d}{d \omega} \frac{d \frac{c^{2} \chi(\zeta) \omega}{\zeta^{2}-\theta^{2}}}{d \theta}\right) \frac{c^{2}}{\zeta^{2}-c^{2}} \frac{d \zeta}{d n}\right\} .
\end{aligned}
$$


E di qui, per (5), applicata al caso delle coordinate in discorso, nell'ipotesi che la superficie $\sigma$ non abbia alcun punto comune colla retta dei punti 0 e 1, sulla quale $Q_{2}$, per $(3)_{i}$ si riduce a 0 , ricaviamo immediatamente:

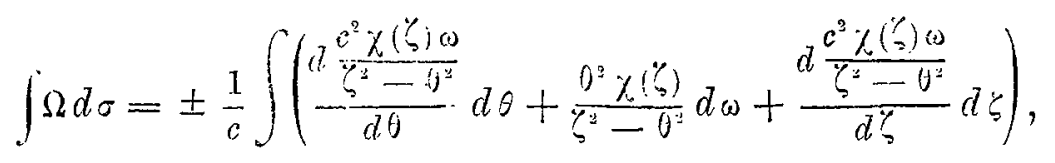

dove, nel secondo membro, l'integrale va esteso al contorno di $\sigma$, e il segno va stabilito colla regola a suo luogo indicata $(\S 5)$.

È poi:

$$
\frac{\theta^{2} \chi(\zeta)}{\zeta^{2}-\theta^{2}}=\frac{\left(\theta^{2}-c^{2}\right) \chi(\zeta)}{\zeta^{2}-\theta^{2}}+\frac{d \frac{c^{3} \chi(\zeta) \omega}{\zeta^{3}-\theta^{3}}}{d(\omega)},
$$

e d'altra parte, colle nostre ipotesi, per la stessa (4):

$$
\int\left(\frac{d \frac{c^{2} \chi(\zeta) \omega}{\zeta^{2}-\theta^{2}}}{d \theta} d \theta+\frac{d^{c^{2} \chi(\zeta) \omega}}{\zeta^{2}-\theta^{2}} d \omega+\frac{d \frac{c^{2} \chi(\zeta) \omega}{\zeta^{2}-\theta^{2}}}{d \zeta} d \zeta\right)=0 .
$$

Quindi finalmente, da (8), nel caso supposto:

$$
\int \Omega d \sigma= \pm \frac{1}{c} \int_{\zeta^{2}-\theta^{2}}^{0^{2}-c^{2}} \chi(\zeta) d \omega
$$

Supposto ora il caso che la retta dei punti 0,1 incontri la superficie $\sigma$, immaginiamo tracciato sulla superficie $\sigma$ un contorno chinso circondante quel punto, e applichiamo la formola ora dedotta alla superficie $\sigma$, avente per contorno questa linea e il contorno di $\sigma$. Se $\int \Omega d \sigma^{\prime}$, col restringersi indefinitamente di questo contorno, avrà un limite indipendente dalla sua forma, assumeremo questo limite come valore di $\int \Omega d$ c corrispondente al caso in discorso.

Ora sarà, distinguendo coll' apice le coordinate dei punti del contorno variabile :

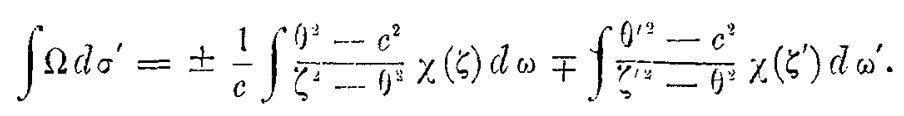

Col restringersi indefinitamente di questo contorno, il primo termino avrà per limite sè stesso. Per cercare il limite del secondo, stabiliamo, in primo luogo, che il senso in cui cresce l'angolo w sia scelto concorde con quello nel quale si suppone percorso il contorno di $\sigma$. In tal caso, $\omega$, in quell'integrale, 
varierà fra 0 e $2 \pi$. Ed essendo la funzione sotto il segno continua in ogni posto, tranne i punti 0 e 1 , fatta eccezione del caso che uno di questi punti cada sulla superficie $\sigma$, sarà:

$$
\lim \frac{1}{c} \int \frac{\theta^{\prime \prime}-c^{\prime 2}}{\zeta^{\prime 2}-\theta^{\prime 2}} \chi(\zeta) d \omega=\frac{2 \pi}{c} \frac{\bar{\theta}^{2}-c^{2}}{\bar{\zeta}^{2}-\bar{\theta}^{2}} \chi(\bar{\zeta}),
$$

dove, i simboli sopralineati indicano le coordinate del punto d'intersezione della superficie $\sigma$ colla retta 0,1 .

Qui bisogna distinguere il caso che la superficie tagli questa retta nel segmento compresu fra 0 e 1 , oppure fuori di questo segmento. Nel primo caso, sarà $\zeta=c$, nel secondo $\bar{\theta}=c ;$ mentre, in ambedue i casi $\bar{\zeta}>\bar{\theta}$. Ne viene che il limite cercato sarà $-\frac{2 \pi}{c} \chi(c)$, nel primo caso, e 0 , nel secondo: per modo che riunendo i varii casi possibili, concludiamo:

$$
\int \Omega d \sigma=\left\{\begin{array}{l} 
\pm \frac{1}{c} \int \frac{\theta^{2}-c^{2}}{\zeta^{2}-\theta^{2}} \chi(\zeta) d \omega \\
\pm \begin{array}{l}
1 \\
c
\end{array} \int \frac{\theta^{2}-c^{2}}{\zeta^{2}-\theta^{2}} \chi(\zeta) d \omega \pm \frac{2 \pi}{c} \chi(c) ;
\end{array}\right\}
$$

dove vale l'espressione superiore e l'inferiore, secondo che la superficie $\sigma$ non è incontrata dal segmento terminato ai punti 0 e 1 , oppure è da esso incontrata.

Per decidere del segno, conformemente alla regola data nel $\S 5$, immagineremo, per un punto qualunque della superficie $\sigma$, descritta la normale alla superficie stessa, e le tangenti alle linee coordinate, ellittica $(\theta)$, circolare $(\omega)$ e iperbolica ( 5 ), nel senso in cui cresce la coordinata rispettiva. E allora si dovrà prendere il segno + , quando il senso della normale e quello in cui si percorre il contorno della superficie $\sigma$, per integrare, stiano, l'uno per rispetto all'altro come il senso della tangente alla linea ellittica $(\theta)$, e quello in cui deve girare un raggio, per portarsi dalla tangente alla linea circolare $(\omega)$ alla tangente alla linea iperbolica $(\zeta)$. Questi, per quanto si è veduto nel $\S 3$, stanno chiaramente, l'uno per rispetto all' altro, come il senso da 1 a 0 della retta passante pei due punti così indicati, e il senso in cui, intorno ad essa, cresce l'angolo $\omega$, comunque venga fissato. In (9) l'angolo $\omega$ è supposto crescere in senso concorde con quello in cui s'immagina percorso il contorno di $\sigma$. Ne viene che il segno + si dovrà adottare, quando dalla retta dei punti 1 e 0 , presa da 1 a 0 , e dalla normale alla superficie $\sigma$, nel punto d'intersezione di 
essa con questa retta, presa nel senso attribuitovi, si veda un punto descrivere il contorno di $\sigma$ nel medesimo senso, e cioè quando le due rette formano fra loro un angolo acuto.

$\S 7$. Le formole precedenti non impongono alla funzione $\psi(\xi)$ alcuna nuova condizione. Nell' ipotesi che le vibrazioni emananti dal centro 1 , conformemente a (1), siano, come le onde luminose, capaci d'interferire, dovrà essere :

$$
\psi\left(\xi+\frac{\lambda}{2}\right)=-\psi(\xi)
$$

dove $\lambda$ indica un certo numero réale. Di qui, per $(6)$ :

$$
\chi\left(\zeta+\frac{\lambda}{4}\right)=-\chi(\zeta)
$$

$\mathrm{E}$ in questa jpotesi, $\int \frac{\theta^{2}-c^{2}}{\zeta^{2}-0^{2}} \chi(\zeta) d \omega$, coll' impiccolire indefinitamente di $\lambda$, ha per limite 0: proprietà, sulla quale riposa l'applicazione delle formole (9) al problema accennato nel $\S 1$.

É facile dimostrare che, nella stessa ipotesi, possiede questa proprietà un integrale della forma $\int \frac{d F}{d \zeta} \chi(\zeta) d \zeta$, supposto il relativo intervallo decomponibile in parti, in ciascuna delle quali $\frac{d F}{d \zeta}$ si mantiene finita, e non cresce o non decresce, nè cambia segno.

Difatti, indichino $\zeta_{0}, \zeta^{\prime}$ i valori estremi d'un siffatto intervallo parziale. Se per avventura, anche $\chi(\zeta)$ non cambierà in esso di segno per (10) sarà:

$$
\left|\zeta^{\prime}-\zeta_{0}\right| \equiv \frac{\lambda}{4}
$$

D'altra parte, rappresentando con $\bar{\zeta}$ un valore appartenente allo stesso intervallo, per un noto teorema, sarà:

$$
\int_{\zeta_{0}}^{\zeta} \frac{d F}{d \zeta} \chi(\zeta) d \zeta=\chi(\bar{\zeta}) \int_{\zeta_{0}}^{\zeta^{\prime}} \frac{d F(\zeta)}{d \zeta} d \zeta=\chi \overline{(\zeta)}\left[F\left(\zeta^{\prime}\right)-F\left(\zeta_{0}\right)\right] .
$$

E la funzione stessa $F(\zeta)$, nelle nostre ipotesi, essendo continua, di qui, per (11), segue immediatamente che, col tendere di $\lambda$ a 0 , l'integrale considerato avrà per limite 0 .

Se poi nell' intervallo $\left(\zeta_{0} \zeta^{\prime}\right) \chi(\zeta)$ cambia una o più volte di segno, per la supposta esistenza della derivata finita di questa funzione, l'intervallo, da un 
egual numero di radici dell' equazione $\%(\zeta)=0$, riuscirà diviso in parti, nelle quali la funzione riceverà valori alternativamente dell'uno e dell'altro segno. Due radici, in ordine di grandezza, successive, avranno per differenza $\frac{\lambda}{4} ;$ e per due valori di $\zeta$, che differiscano egualmente da ciascuna, $\chi(\zeta)$ riceverà valori assoluti eguali. Da ciò, indicando con $a_{r-1}$ il valore assoluto di

$$
\int \frac{d F}{d \zeta} \chi(\zeta) d \zeta
$$

esteso all' $r^{m a}$ di quelle parti, supposte $n$, per modo che:

$$
\int_{\zeta_{0}}^{\zeta^{\prime}} \frac{d F}{d \zeta} \chi(\zeta) d \zeta= \pm\left(a_{0}-a_{1}+a_{2}-\cdots \pm a_{n-1}\right),
$$

si dedurrà assai facilmente che l'integrale considerato, preso, a seconda del caso, col segno + 0 -, sarì compreso fra $-a_{1}$ e $a_{0}$, se nell'intervallo $\left(\zeta_{0}, \zeta^{\prime}\right)$ $\chi(\zeta)$ non è crescente, e, se non è decrescente, fra $-a_{n-2}$ e $a_{n-1}$. D'altra parte, pel teorema testè ricordato, indicando con $\bar{\zeta}$ un valore di $\zeta$ appartenente all'intervallo parziale $\left(\zeta_{r-1}, \zeta_{r}\right)$,

$$
\int_{\zeta r-1}^{\zeta^{r}} \frac{d F(\zeta)}{d \zeta} \chi(\zeta)=\chi(\bar{\zeta})\left[F\left(\zeta_{r}\right)-F\left(\zeta_{r-1}\right)\right]
$$

dove $\left|\zeta_{r}-\zeta_{r-1}\right| ₹ \frac{\lambda}{4}$ : per modo che, coll'impiccolire di $\lambda, a_{0}, a_{1}, a_{r-2}, a_{r-1}$ hanno per limite 0 . Per quanto precede, lo stesso limite avrà il nostro integrale $(*)$.

Ciò premesso, ci basterà mostrare che l'integrale $\int \frac{\theta^{2}-c^{2}}{\zeta^{2}-\theta^{2}} \chi(\zeta) d \omega$, con opportune limitazioni, si può ridurre al tipo ora considerato. E questo si vede facilmente. Supposto infatti che il contorno di $\sigma$, al quale l'integrale va esteso, si possa decomporre in tante parti, tali che, presa una qualunque di esse, ad ogni valore di $\zeta$ appartencnte ad un certo intervallo, corrisponda uno ed uno solo de' suoi punti, per modo che le coordinate $\omega$ e $\theta$ de' punti stessi siano, in quell' intervallo, funzioni di $\zeta$ : e supposte queste funzioni continue e finite, e $\omega$ dotata delle derivate per rispetto a $\zeta$, continua e finita: indicando con $\zeta_{0}, \zeta^{\prime}$

(*) Questa proposizione serve anche all analisi di KukchHoff. E la dimostrazione è conforme alla traccia ch'egli ne dà, nella Memoria più volte citata. 
gli estremi d'un intervallo siffatto, e con $\omega_{1}$, w' i corrispondenti valori di $\omega$, sarà, in primo luogo,

$$
\int_{\omega 0}^{\omega_{0} \theta^{2}} \frac{\theta^{2}-e^{2}}{\zeta^{2}-\theta^{2}} \chi(\zeta) d \omega=\int_{\zeta_{0}}^{\zeta} \frac{0^{3}-r^{2}}{\zeta^{2}-\theta^{2}} \chi(\zeta) \frac{d \omega}{d \zeta} d \zeta .
$$

Sarà poi $\frac{\theta^{2}-c^{2}}{\zeta^{2}-\theta^{3}} \frac{d \omega}{d \zeta}$ nell'intervallo $\left(\zeta_{0}, \zeta\right)$, atta all' integrazione definita; per modo che posto:

$$
\frac{\theta^{2}-c^{2}}{\zeta^{2}-\theta^{2}} \frac{d \omega}{d \zeta}=\frac{d F(\zeta)}{d \zeta}
$$

la funzione $F(\zeta)$, in quell' intervallo csisterà realmente, e sarà finita. E por mezzo di questa relazione:

$$
\int_{\omega_{0}}^{\omega^{\prime} \theta^{2}-c^{2}} \frac{\zeta^{2}-\theta^{2}}{\zeta^{2}} \chi(\zeta) d \omega=\int_{\zeta_{0}}^{\zeta^{\prime}} \frac{d F^{\prime}(\zeta)}{d \zeta} \chi(\zeta) d \zeta .
$$

$\S 8$. Per $(6)$, indicando per un momento con $r_{01}$ la mutua distanza dei punti 0 e 1 , per modo che $c=\frac{r_{01}}{2}$ :

ed avendo posto:

$$
\frac{2 \pi}{c} \chi(c)=4 \pi \frac{\dot{\varphi}\left(r_{01}-a t\right)}{r_{01}}
$$

$$
\varphi(t, x, y, z)=\frac{\psi\left(r_{1}-a t\right)}{r_{1}}
$$

colla serittura convenuta:

$$
\frac{2 \pi}{c} \chi(c)=4 \pi \varphi_{0}(t)
$$

Riprendiamo ora le (9). Da esse, e dai risultati del precedente paragrafo, concludiamo che, supposto:

$$
\psi(\xi+\lambda)=-\psi(\xi)
$$

ipotesi corrispondente a quella che le onde emananti dal centro 1 siano luminose, e, più generalmente, capaci d'interferire, sarà:

$$
\int \Omega d \sigma=\left\{\begin{array}{l}
\Lambda \\
\Lambda \pm 4 \pi \varphi_{0}(t)
\end{array}\right.
$$

dove la scelta del valore e del segno va fatta coi criteri indicati per le (9): e $\Lambda$ rappresenta una quantità, che, coll'impiccolire indefinitamente di $\lambda$, ha per limite 0 . 
III.

A complemento di ciò che precede, credo opportuno aggiungere la seguente deduzione del teorema di Stores.

Partiamo dalla considerazione di una linea rientrante, che supporremo non tagliare sè stessa, e indicando con $q_{1}, q_{2}, q_{3}$ le coordinate dei punti di una regione dello spazio comprendente quella linea, comunque definito, e con $U_{1}, U_{2}, U_{3}$ tre funzioni di esse, supponiamo che nella linea in discorso queste funzioni siano monodrome, continue e finite. In tal caso

$$
\int\left(U_{1} d q_{1}+U_{2} d q_{2}+U_{3} d q_{3}\right)
$$

esteso alla linea considerata, avrà un valore determinato e finito.

Ora supponiamo che esista una superficie, avente per contorno la linea precedente, e assoggettata a quelle condizioni che emergeranno dalle seguenti ipotesi, tra cui a quella d'avere in ogni punto jl piano tangente, sulla quale le funzioni $U_{1}, U_{2}, U_{3}$ siano monodrome, continue e finite, e ammettano i coefficienti differenziali per rispetto a ciascuna variabile, finiti.

Fissati due punti, $A$ e $B$, della linea considerata, che la divideranno in due parti, $A C B$ e $A C^{\prime} B$, immaginiamo nella superficie suddetta una successione di linee, di cui $A C B$ e $A C^{\prime} B$ siano gl'individui estremi, le quali non tagliıo sè stesse, nè alcun altro individuo della successione medesima, e tali che, per ogni punto della superficie ne passi una, e, con essa, un'altra, colle stesse proprietà, all'infuori della prima. Per ogni punto della superficie passerà così una coppia determinata di linee, dell'una e dell'altra successione: o reciprocamente ogni coppia siffatta determinerà un punto della superficie.

Ciò torna a concepire le coordinate $q_{1}, q_{2}, q_{3}$ dei punti della superficie come funzioni di due parametri opportuni, $u$ e $v$, corrispondenti alle due successioni di linee: in modo che, fissato uno dei due parametri, riesce fissato una linea d'una delle due successioni, lungo la quale varia il parametro rimanente. Indicheremo le linee delle due successioni col simbolo del parametro lungo di esse variabile: e supporremo che $u$ varii lungo le linee della prima successione, che perciò chiameremo anche linee $u$.

Ammetteremo poi che le due successioni di linee si possano scegliere in modo che le corrispondenti funzioni $q_{1}, q_{2}, q_{3}$ di $u$ e $v$ ammettano la derivata per rispetto a ciascun parametro, e queste derivate, la derivata per ri- 
spetto all' altro pirametro, per modo, stabilendo d'indicare con $\partial$ e $\delta$ rispettivamente $\mathrm{i}$ differenziali per rispetto a $u$ e a $v$, esistano $\partial q_{i}, \delta q_{i}$ e $\partial \delta q_{i}=\delta \partial q_{i}$ $(i=1,2,3)$. e sarà :

$\mathrm{Ne}$ verrà, in virtù delle precedenti ipotesi, che esisteranno $\partial U_{i}$ e $\partial U_{i}$,

$$
\left.\begin{array}{l}
\partial U_{i}=\frac{d U_{i}}{d q_{1}} \partial q_{1}+\frac{d U_{i}}{d q_{2}} \partial q_{2}+\frac{d U_{i}}{d q_{3}} \partial q_{3} \\
\partial U_{i}=\frac{d U_{i}}{d q_{1}} \partial q_{1}+\frac{d U_{i}}{d \eta_{2}} \partial q_{2}+\frac{d U_{i}}{d q_{3}} \partial q_{3} .
\end{array}\right\}
$$

Inoltre, esteso ad ngni $u$, esisterà.

$$
\int\left(U_{1} \partial q_{1}+U_{2} \partial q_{2}+U_{3} \partial q_{3}\right)
$$

e sarì una funzione di $v$, di cui esisterà il differenziale:

$$
\delta \int\left(U_{1} \partial q_{1}+U_{2} \partial q_{2}+U_{3} \partial q_{3}\right)
$$

Di questo differenziale ci giova trovare una forma opportuna. Perciò osserviamo che, per teoremi noti, nelle nostre ipotesi, sarà:

$$
\begin{aligned}
\delta \int U_{i} \partial q_{i} & =\int \partial\left(U_{i} \partial q_{i}\right)=\int \delta U_{i} \partial q_{i}+\int U_{i} \partial \partial q_{i} \\
\int U_{i} \partial \partial q_{i} & =\int U_{i} \partial \partial q_{i}, \\
\int U_{i} \partial \partial q_{i} & =-\int \partial U_{i} \partial q_{i},
\end{aligned}
$$

poichè nei punti limiti dell' integrazione $\delta^{\prime} q_{i}$ è nullo (le linee $u$ avendo per estremi comuni i punti $A$ e $B$ del contorno). Quindi:

$$
\delta \int U_{i} \partial q_{i}=\int\left(\delta U_{i} \partial q_{i}-\partial U_{i} \delta q_{i}\right)
$$

E di qui, impiegando le (2), deduciamo, dopo ovvie riduzioni, da (3):

$$
\begin{gathered}
\delta \int\left(U_{1} \partial q_{1}+U_{2} \partial q_{2}+U_{3} \partial q_{3}\right) \\
=\int\left\{\left(\frac{d U_{3}}{d q_{2}}-\frac{d U_{2}}{d q_{3}}\right)\left(\partial q_{2} \partial q_{3}-\partial q_{3} \partial q_{2}\right)+\left(\frac{d U_{1}}{d q_{3}}-\frac{d U_{3}}{d q_{1}}\right)\left(\partial q_{3} \partial q_{1}-\delta q_{1} \partial q_{3}\right)+\right. \\
\left.+\left(\frac{d U_{2}}{d q_{1}}-\frac{d U_{1}}{d q_{2}}\right)\left(\delta q_{1} \partial q_{2}-\partial q_{2} \partial q_{1}\right)\right\}
\end{gathered}
$$

che è il risultato voluto. 
D'altra parte, l'integrale (1) esteso alla linea rientrante considerata, e cioè al contorno chiuso della superficie in discorso, è, se vogliamo, la differenza dei valori che riceve l'integrale (3), esteso alle due parti in cui essa linea vien divisa dai punti $A$ e $B$ : e precisamente, se la linea rientrante si suppone percorsa nel senso indicato dalla successione delle lettere $A C B C^{\prime} A$, eguale al valore corrispondente alla linea $A C B$, meno quello che corrisponde alla linea $A C^{\prime} B$.

La stessa differenza è anche espressa da:

$$
\int \delta \int\left(U_{1} \partial q_{1}+U_{2} \partial q_{2}+U_{3} \partial q_{3}\right)
$$

dove la seconda integrazione $\mathrm{s}^{\prime}$ intende aver per limiti $\mathrm{i}$ valori di $v$ corrispondenti alle linee estreme $A C^{\prime} B$ e $A C B$ : e cioè estesa all'intervallo che abbraccia $\mathrm{i}$ valori di $v$ corrispondenti a tutte le linee della successione di cui quelle costituiscono gl'individui estremi.

Quindi, per la precedente relazione, collo stesso significato di ciascun segno,

$$
\begin{gathered}
\int\left(U_{1} d q_{1}+U_{2} d q_{2}+U_{3} d q_{3}\right)= \\
=\iint\left\{\left(\frac{d U_{3}}{d q_{2}}-\frac{d U_{2}}{d q_{3}}\right)\left(\delta q_{2} \partial q_{3}-\partial q_{3} \partial q_{2}\right)+\left(\frac{d U_{1}}{d q_{3}}-\frac{d U_{3}}{d q_{1}}\right)\left(\delta q_{3} \partial q_{1}-\delta q_{1} \partial q_{3}\right)+\right. \\
\left.+\left(\frac{d U_{2}}{d q_{1}}-\frac{d U_{1}}{d q_{2}}\right)\left(\partial q_{1} \partial q_{2}-\partial q_{2} \partial q_{1}\right)\right\} .
\end{gathered}
$$

Questa è una forma del teorema di STokes, applicabile a qualunque specie di coordinate curvilinee. È facile passare da essa alla nota forma, relativa a coordinate ortogonali.

A tal fine, indicando con $d s$ il differenziale di un arco di linea qualunque, corrispondente ai differenziali $d q_{1}, d q_{2}, d q_{3}$ delle coordinate $q_{1}, q_{2}, q_{3}$, sia:

$$
d s^{2}=Q_{1}^{2} d q_{1}^{2}+Q_{2}^{2} d q_{2}^{2}+Q_{3}^{2} d q_{3}^{2}
$$

$u, v$ rappresentino gli archi delle linee così segnate, terminati al punto $\left(q_{1}, q_{2}, q_{3}\right):$ e, nell'jpotesi che il contorno di $\sigma$ si debba percorrere nel senso $A C B C^{\prime} A$, sia $u$ contato da $A$, e $v$ dal punto in cui la rispettiva linea incontra $A C^{\prime} B$ : per modo che indicando $\partial u$, $o v$ i differenziali degli stessi archi corrispondenti ai differenziali $\partial q_{i}, \delta q_{i}$ di $q_{i}(i=1,2,3)$, quei differenziali siano positivi, quando si passa dal punto $\left(q_{i}\right)$ ai punti $\left(q_{i}+\partial q_{i}\right)$ e $\left(q_{i}+\dot{\delta} q_{i}\right)$ camminando rispettivamente lungo $u$ da $A$ verso $B$, e lungo $v$ da $A C^{\prime} B$ verso $A C B$; indichi $(u, v)$ l'angolo formato dalle tangenti alle due linee $u$ e $v$, passanti pel punto $\left(q_{i}\right)$, volte nel senso in cui crescono gli archi $u, v$; finalmente, 
concependo per un momento, le coordinate di un punto qualunque dello spazio come funzioni della distanza normale $n$ del punto dalla superficie considerata (e di due altri parametri, come potrebbero essere $u$ e $v$ ) $\left(^{*}\right), d q_{1}, d q_{2}, d q_{3}$ ne rappresentino i differenziali, corrispondenti al differenziale $d n$ di questa distanza: e precisamente $i$ differenziali cosi definiti nello stesso punto $\left(q_{1}, q_{*}, \gamma_{3}\right)$ della superficie, a cui si riferiscono i precedenti valori delle variabili.

In tal caso sarà, per formole ben note,

$$
\frac{Q_{2} \delta q_{2}}{\delta v} \frac{Q_{3} \hat{\partial} q_{3}}{\hat{\partial} u}-\frac{Q_{3} \dot{\delta} q_{3}}{\delta v} \frac{Q_{2} \hat{\partial} q_{9}}{\partial u}= \pm \frac{Q_{1} d q_{1}}{d u} \sin (u, v)
$$

dove $Q_{1}, Q_{3}, Q_{3}$ indicano valori assoluti: e si deve prendere il segno + , quando l'angolo $(u, v)$ non sia maggiore d'un retto, e due osservatori, coi piedi nel punto considerato della superficie, e disposti lungo la normale, volta nel senso in cui cresce $n$, e lungo la tangente alla linea $q_{1}$, nel senso in cui cresce la relativa coordinata, velano girare in senso concorde, il primo, un raggio che passa, descrivendo l'angolo predetto, dalla tangente alla linea $v$ alla tangente alla linea $u$, volte nel senso in cui rispettivamente crescono $v$ e $u$, e jl secondo, un raggio che passa dalla tangente alla linea $q_{2}$ alla tangente alla linea $q_{3}$, volte, l'una e l'altra, nel senso in cui cresce la rispettiva coordinata.

Dalla formola precedente, nell'ipotesi che $Q_{1}, Q_{2}$, nel punto considerato, abbiano un valore superiore a 0 , si ricava, colla stessa regola per la scelta del segno,

$$
\delta q_{2} \partial q_{3}-\delta q_{3} \partial q_{2}= \pm \frac{Q_{1}}{Q_{3} Q_{3}} \frac{d q_{1}}{d n} \sin (u, v) \partial u \delta v
$$

D'altra parte, indicando con $d \sigma$ l'area del quadrilatero curvo limitato dalle due coppie di lince $u$ e $v$, l'una passante pel punto considerato, e l'altra corrispondente a quegli aumenti di questi parametri a cui si riferiscono i difforenziali delle nostre formole: supposto che questi aumenti tondano a 0 : sarà, colla differenza di un evanescente d'ordine superiore a quello di $\partial u \partial v$ o di $d \sigma$,

$$
d \sigma= \pm \sin (u, v) \partial u v^{2} v
$$

dove il segno + va preso nell'ipotesi che l'angolo $(u, v)$ non superi un retto, c. $\partial u, j v$ abbiano lo stesso segno.

$\mathrm{Ne}$ viene, colla differenza d'un evanescente dello stesso ordine del precedente,

$$
\delta q_{2} \partial q_{3}-\partial q_{3} \partial q_{2}= \pm \frac{Q_{1}}{Q_{2} Q_{3}} \frac{d q_{1}}{d n} d \sigma
$$

dove la scelta del segno non dipende più dall' angolo $(u, v)$.

$\left.{ }^{*}\right)$ Cfr. Beltrani: Intomo al alouni punti della teorica del potenaiale. Mom. dell'Acc. di Bologna, 1878. 
E per (4), supposto che, in ogni punto della superficie, $Q_{1}, Q_{2}, Q_{3}$ ricevano valori superiori a 0 ,

$$
\left.\begin{array}{c}
\int\left(U_{1} d q_{1}+U_{2} d q_{2}+U_{3} d q_{3}\right) \\
= \pm \int\left(\frac{d U_{3}}{d q_{2}}-\frac{d U_{2}}{d q_{3}}\right) \frac{Q_{1}}{Q_{2} Q_{3}} \frac{d q_{1}}{d n}+\left(\frac{d U_{1}}{d q_{3}}-\frac{d U_{3}}{d q_{1}}\right) \frac{Q_{2}}{Q_{3} Q_{1}} \frac{d q_{2}}{d n}+ \\
\left.+\left(\frac{d U_{2}}{d q_{1}}-\frac{d U_{1}}{d q_{2}}\right) \frac{Q_{3}}{Q_{1} Q_{2}} \frac{d q_{3}}{d n}\right\} d \sigma .
\end{array}\right\}
$$

Nell'ipotesi che, nell'integrale del primo membro, il contorno della superficie venga percorso nel senso $A C B C^{\prime} A$, nel secondo, per integrare per rispetto a $u$ e a $v$, si percorreranno le relative linee rispeltivamente da $A$ verso $B$, e da $A C^{\prime} B$ verso $A C B$. Quindi, per le nostre precedenti convenzioni, in primo luogo ai $\partial q_{i}$, $\partial q_{i}(i=1,2,3)$ implicati dall'integrale del secondo membro di (4) corrisponderanno valori positivi di $\partial u$, ov $v$ e, in secondo luogo, il senso in cui deve girare un raggio intorno ad un punto della superficie, per trasportarsi, dalla tangente alla linea $v$, alla tangente alla linea $u$, volte nel senso in cui questi parametri crescono, sarà concorde con quello in cui, nell'integrale del primo membro, si suppone percorso il contorno della superficie. Si conclude da ciò che nella (5) si dovrà prendere il segno + , quando due osservatori, coi piedi in un punto della superficie, e disposti, l'uno secondo la normale in esso punto, nel senso attribuitole nel secondo membro per differenziare, e l'altro secondo la tangente alla linea $q_{1}$ passante pel punto, volta nel senso in cui cresce la coordinata $q_{1}$, vedano girare in senso concorde, il primo, un punto, che descrive il contorno della superficie, nel senso assunto nel prino membro per integrare, e il secondo, un raggio che, dalla tangente alla linea $q_{2}$ passante pel punto, si trasporta alla tangente alla linea $q_{3}$ passante per lo stesso punto, l'una e l'altra volta nel senso in cui cresce la relativa coordinata.

Questa regola è svincolata da ogni considerazione delle linee coordinate, che abbiamo immaginato tracciate sulla superficie $\sigma$, per arrivare alla formola (5). E occorre appena ricordare che, mantenendo le debite ipotesi sulle funzioni che figurano in essa, il valore dell' integrale di superficie sarà indipendente da ogni particolar modo di decomposizione della superficie medesima: per modo che, neppure per mezzo di $d \sigma$, rimane effettivamente traccia delle linee suddette. 
IV.

$\S 1$. Venendo infine all' accennato problema d'ottica, suppongasi che un corpo estraneo sia immerso in un mezzo dove si propaga una serie di onde sferiche emananti da un centro: e la superficie di questo corpo ammetta in ogni punto il piano tangente. Questo sarà un corpo nero, quando attraverso nessun punto della sua superficie si propagheranno onde, dal piano tangente nel punto, verso un altro piano ad esso parallelo, e, in un intorno sufficientemente piccolo del punto, esterno alla superficie. Tale è in sostanza la definizione di KirchHofF.

Considereremo particolarmente il caso che la superficie del corpo in discorso, da ogni segante uscente dal centro delle onde sia incontrata in due punti, e in nessun altro, e toccata da una superficie conica avente il vertice nel centro medesimo, la quale sarà il limite fra le rette, uscenti dallo stesso punto, che segano e non segano la superficie del corpo considerato. E questo corpo lo supporremo immerso in un mezzo esternamente indefinito, e internamente limitato dalla sua superficie.

Ammesso che al presente caso si possa applicare la formola (12) di I, le funzioni $\varphi(t, x, y, z)$ e $f(t, x, y, z)$ dei punti della superficie $\sigma$ del corpo debbono concepirsi scelte in modo che si verifichi la proprietà assunta come definizione d'un corpo nero.

Distinguiamo le due calotte in cui tale superficie è decomposta dalla linea di contatto colla superficie conica avente per vertice il centro delle onde, o punto 1: la prima volta verso questo punto, e l'altra opposta. Il termine $\varphi_{0}^{*}(t)$ del secondo membro della formola ricordata corrisponde ad una successione di onde sferiche che, supposto il punto 0 sulla prima calotta, in un intorno abbastanza piccolo di esso, si propagano dall'esterno della superficie verso il piano tangente ad essa nel punto, mentre il contrario avviene, nell'ipotesi che il punto 0 sia sulla seconda calotta.

Per conseguenza, le due suddette funzioni dei punti della superficie $\sigma$ debbono concepirsi scelte in modo che, componendo questo moto ondulatorio con quello che corrisponde al termine $\frac{1}{4 \pi} \int \Omega d \sigma$ (cfr. I, $\S 12$ ), - per cui valore, nel punto $(x, y, z)$ di $\sigma$ deve concepirsene assunto il limite col tendere di $\left(x_{0} y_{0} z_{0}\right)$ allo stesso punto - nel moto risultante, cessino di esistere effettivamente onde sensibili, che si propagano in quel modo per rispetto ai punti della seconda calotta, e non ne nascano, che si propaghino allo stesso modo per rispetto ai punti della prima. 
§ 2. Richiamando le circostanze speciali alle onde luminose (cfr. $§ 7$ di II), conveniamo che ogni grandezza che tende a 0 colla lunghezza d'onda $\lambda$ debba reputarsi come insensibile $\left({ }^{*}\right)$.

In tale ipotesi alla relazione più volte rammentata si dovrà sostituire:

$$
\varphi_{0}(t)=\varphi_{0}^{*}(t)+\frac{1}{4 \pi} \int\left\{\frac{\partial \frac{\varphi^{\prime}\left(t-\frac{r}{a}\right)}{r}}{\partial n}-\frac{f\left(t-\frac{r}{a}\right)}{r}\right\} d \sigma,
$$

dove $\varphi^{\prime}(t), f^{\prime}(t)$ indicano due funzioni di $t$ e delle coordinate dei punti della superficie $\sigma$, che, sopra di essa, nei singoli posti, ricevono valori differenti da quelli che vi ricevono $\varphi(t)$ e $f(t)=\frac{\partial \varphi(t)}{d n}$, rispettivamente, per una quantità evanescente colla lunghezza d'onda.

E lo scopo spiegato nel paragrafo precedente si dovrà considerare come conseguito, quando $\varphi_{0}(t)$, in virtù di quella relazione, riceva, sopra la calotta $\sigma$ affacciata al punto 1 , valori differenti da quelli di $\varphi_{0}(t)$ per una quantità evanescente colla lunghezza d'onda, e, sopra l'altra, aventi essi stessi quest'ultima proprietà.

Dovendo poi questi valori sopra ambedue le calotte differire per una quantità evanescente colla lunghezza d'onda da quelli attribuiti a $\varphi^{\prime}(t)$, e una differenza analoga sussistere tra $\frac{d \varphi(t)}{d n}$ e $f^{\prime}(t)$, si presenta spontanea su la prima calotta l'ipotesi:

e su la seconda:

$$
\left.\begin{array}{c}
\varphi^{\prime}(t)=\varphi^{*}(t), \quad f^{\prime}(t)=\frac{d \varphi^{*}(t)}{d n}, \\
\varphi^{\prime}(t)=0, \quad f^{\prime}(t)=0 .
\end{array}\right\}
$$

Introducendo queste ipotesi nella (1), e richiamando la (11) di II, ne dedurremo immediatamente:

$$
\varphi_{0}(t)=\left\{\begin{array}{l}
\varphi_{0}^{*}(t)+\Lambda \\
\Lambda,
\end{array}\right\}
$$

dove $\Lambda$ rappresenta una quantità evanescente colla lunghezza d'onda: e va

(*) Giova notare che, dal punto di vista dell accordo fra la teoria e l'esperienza, questa condizione non sarà sufficiente perchè la grandezza si possa reputare trascurabile. Perciò occorrerà di più ch'essa cada nei limiti attribuiti agli errori d'osservazione, come si sup. pone aver lnogo, nell'attuale questione, per la lunghezza d'onda. 
preso il valore superiore o l'inferiore, secondo che la retta terminata ai punti 0 e 1 non attraversa, oppure attraversa la calotta della superficie $\sigma$ volta verso il punto 1 , cioè la superficie stessa: ossia, secondo che il punto 0 si suppone estermo alla regione limitata dal cono arente il vertice nel punto 1 , e tangente alla superficie del corpo, e dalla calotta di questa superficie non prospiciente il punto 1 , oppure interno alla regione medesima.

$\mathrm{Ne}$ viene che, coll'avricinarsi del punto 0 ad un punto della calotta prospiciente il punto 1 , o della calotta opposta, $\varphi_{0}(t)$, in virtu di (1), tenderà rispettivamente a $\varphi_{0}{ }^{*}(t)+\Lambda$ o a $\Lambda$, che differiscono per una quantità evanescente colla lunghezza d'onda dai relativi valori di $\varphi^{\prime}(t)$ : mentre allo stesso modo, su tutta la superficie, differirà $\frac{d \varphi^{*}(t)}{d n}$ da $f^{\prime}(t)$. Ciò verifica a posteriori la legittimità della relazione (1) colle ipotesi (2): dalla quale relazione inoltre $\varphi_{0}(t)$ è definito in ogni posto dalla regione interna e esterna a quella superficie conica, e riesce in ambedue le regioni una funzione che soddisfa all'equazione differenziale (1), e alla condizione del $\S 9$ di I, e soltanto nella superficie conica stessa presenta una discontinuità $(*)$.

Per la nostra convenzione, la (3) equivale sensibilmente, colla stessa corrispondenza, a

$$
\varphi_{0}(t)=\left\{\begin{array}{l}
\varphi_{0}^{*}(t) \\
0 .
\end{array}\right.
$$

E per conseguenza, nella regione interna alla superficie conica suddetta il mezzo resterà sensibilmente in quiete, mentre fuori di essa si moverà sensibilmente, come se il rorpo estraneo non esistesse. In altre parole, colle limitazioni e le ipotesi ammesse, la precedente teoria ci conduce alla conclusione che il corpo nero projetterà nello spazio il cono d'ombra, come prescindendo dai fenomeni di diffrazione, corrispondenti ad estensioni dello stesso ordine di piccolezza della lunghezza d'onda, verifica l'esperienza.

Caste'lo Valtravag!ia, ottobre 1887.

(*) Si riconoscerì nella discontinuità indicata dalle (2), lungo la linea di congiunzione delle due calotte di $\sigma$, la ragione per cui, colle stesse ipotesi, l'intcgrale che figura in (1) non fornisce per limite di $\varphi(t)$ e di $\frac{d \varphi(t)}{d n}$, col tendere del punto corricpondente ad un punto della superficie, $\varsigma^{\prime}(t)$ e $t^{\prime}(t)$, come vorrebhe la (12) di I. 